\title{
ON THE NON-STEADY MOTIONS OF A RIGID BODY IN AN IDEAL FLUID*
}

\author{
BY \\ G. W. MORGAN \\ Brown University
}

1. Introduction and summary. If a rigid body moves in vacuum under the action of a single external force which has a constant direction but possibly varying magnitude and whose line of action passes through the center of mass of the body, the body will maintain a pure translatory motion parallel to the direction of the force. If the body is surrounded by a fluid, (in the following assumed to be inviscid and incompressible), such a motion will, in general, no longer be possible. The following question arises: Is there a point such that a body acted on by a force which has constant direction and whose line of action passes through this point, will move in a straight line parallel to the force without rotation? The investigation presented below gives the following answers to this question.

a) There is no such point in the most general case.

b) If the shape of the body satisfies certain conditions, then there exist three axes, each associated with the body and with one of three directions relative to the body, (the so-called directions of permanent translation)**, as well as with the density of the fluid, such that, if a force of constant or varying magnitude acts on the body with its line of action along one of the axes and if there are no other external forces, the body will move with pure translation parallel to the force and axis. The conditions which the body shape must satisfy are tantamount to certain symmetry requirements.

c) In the case of plane motion (without circulation), i.e. if the body is an infinite cylinder with its generators perpendicular to its direction of motion, there exists a unique point (for a fixed fluid density $\rho$ ) such that a body, whose motion is due entirely to an external force which acts parallel to one of the two directions of permanent translation with its line of action passing through the point, will move without rotation parallel to the force. This point might be referred to as the "apparent center of mass" of the body corresponding to the given fluid.

d) For a body of revolution there also exists an apparent center of mass.

Inasmuch as these considerations concern a problem in classical hydrodynamics, one might expect to find them in the standard literature on the subject, but, to his surprise, the author was unable to do so.

Our plan of procedure is to set up the expressions for the kinetic energy of the body and the fluid and then to investigate the equations of motion of the body in terms of the kinetic energies.

2. Kinetic energy of the body. Consider a system of rectangular Cartesian axes fixed to the body with origin at $O$. Using tensor notation, the motion of the body can be described in terms of the translational velocity $U_{i}$ of the origin and rotation with angular

\footnotetext{
*Received Aug. 19, 1953. This paper represents a slightly condensed version of a report prepared under Contract N7onr-35810 between the Office of Naval Research and Brown University.

${ }^{* *}$ Lamb, Hydrodynamics Ch. VI.
} 
velocity $\omega_{i}$ about the origin. If $x_{i}$ denotes the position vector of a point of the body with respect to $O$, then the kinetic energy of the body, $T_{B}$, is given by

$$
2 T_{B}=\int_{B}\left(U_{i}+\epsilon_{i j k} \omega_{i} x_{k}\right)\left(U_{i}+\epsilon_{i l_{n} \omega_{l}} x_{n}\right) \sigma d \tau,
$$

where $\sigma$ is the density of the body, $\epsilon_{i j k}$ is the alternating tensor, and the volume integration extends over the entire body $B$. Since

this can be written as

$$
\epsilon_{i j k} \epsilon_{i l n} x_{k} x_{n}=\left(\delta_{j l} x_{k} x_{k}-x_{j} x_{l}\right),
$$

$$
2 T_{B}=U_{i} C_{i} \int_{B} \sigma d \tau+L_{i} \omega_{i} \epsilon_{i j k} \int_{B} \sigma x_{k} d \tau+\omega_{i} U_{i} \epsilon_{i j k} \int_{B} \sigma x_{k} d \tau+\omega_{i} \omega_{i} I_{i s},
$$

where $I_{i j}$ is the inertia tensor of the body and is given by

$$
I_{i j}=\int_{B} \sigma\left(\delta_{i j} x_{k} x_{k}-x_{i} x_{j}\right) d \tau .
$$

We note that this expression involves ten independent constants:

$$
\begin{aligned}
& \text { the mass of the body } \int_{B} \sigma d \tau=M, \\
& \text { the three moments } \int_{B} \sigma x_{k} d \tau=M x_{k}^{*},
\end{aligned}
$$

where $x_{k}^{*}$ denotes the position of the center of mass, and the six independent components of the symmetric inertia tensor $I_{i i}$.

At this point it is convenient to introduce a new notation. Let

$$
\begin{array}{lll}
V_{1}=U_{1}, & V_{2}=U_{2}, & V_{3}=U_{3}, \\
V_{4}=\omega_{1}, & V_{5}=\omega_{2}, & V_{6}=\omega_{3} .
\end{array}
$$

By $V_{\alpha}$ we shall mean any of the six components $V_{1}$ to $V_{6}$ with $\alpha$ ranging from 1 to 6 . We use Greek subscripts to differentiate the present notation from tensor notation. Also, supposing the summations implied in the expression (2) to be carried out and the $U_{i}$ and $\omega_{i}$ to be replaced by the appropriate $V_{\alpha}$, let $M_{\alpha \beta}$ be the coefficient of the term in $V_{\alpha} V_{\beta}$. The 36 quantities $M_{\alpha \beta}$ then have the following values:

$$
\begin{aligned}
& M_{11}=M_{22}=M_{33}=M, \\
& M_{12}=M_{21}=M_{13}=M_{31}=M_{23}=M_{32}=0, \\
& M_{14}=M_{41}=M_{25}=M_{52}=M_{36}=M_{63}=0, \\
& M_{15}=M_{51}=-M_{24}=-M_{42}=M x_{3}^{*}, \\
& M_{16}=M_{61}=-M_{34}=-M_{43}=-M x_{2}^{*}, \\
& M_{25}=M_{62}=-M_{35}=-M_{53}=M x_{1}^{*}, \\
& M_{44}=I_{11}, \quad M_{55}=I_{22}, \quad M_{66}=I_{33}, \\
& M_{45}=I_{12}, \quad M_{54}=I_{21}, \quad \text { hence } M_{45}=M_{54}, \\
& M_{46}=I_{13}, \quad M_{64}=I_{31}, \quad \text { hence } M_{46}=M_{64}, \\
& M_{56}=I_{23}, \quad M_{65}=I_{32}, \quad \text { hence } M_{56}=M_{65} .
\end{aligned}
$$


We can now write expression (2) in the compact form

$$
2 T_{B}=\sum_{\alpha, \beta=1}^{6} V_{\alpha} V_{\beta} M_{\alpha \beta} \text {. }
$$

3. Kinetic energy of the fluid. Since the motion of the fluid is assumed to be entirely due to that of the solid, it is irrotational. Its velocity potential $\Phi$ is of the form

$$
\Phi=\sum_{\alpha=1}^{6} V_{\alpha} \varphi_{\alpha}
$$

where the $\varphi_{\alpha}$ are functions of the coordinates $x_{1}, x_{2}, x_{3}$ depending solely on the shape and position of the solid surface relative to the coordinate axes fixed in the body. They represent the velocity potentials due to the motion of the body with unit translational or rotational velocity with respect to the appropriate axes.* The kinetic energy of the fluid, $T_{F}$, is given by

$$
2 T_{F}=\rho \int_{S} \Phi \frac{\partial \Phi}{\partial n} d S
$$

where $\rho$ is the fluid density, $\partial / \partial n$ denotes differentiation with respect to the outwardly directed normal to the surface $S$, and the integration extends over the surface $S$ of the body.

Substitution of (8) into (9) gives

$$
2 T_{F}=\sum_{\alpha z \beta}\left(V_{\alpha} V_{\beta} \rho \int_{S} \varphi_{\alpha} \frac{\partial \varphi_{\beta}}{\partial n} d S\right)
$$

Each integral

$$
\int_{S} \varphi_{\alpha} \frac{\partial \varphi_{\beta}}{\partial n} d S
$$

is a constant determined exclusively by the shape and position of the body surface $S$ relative to the coordinate axes. Hence, setting

$$
\rho \int_{S} \varphi_{\alpha} \frac{\partial \varphi_{\beta}}{\partial n} d S=F_{\alpha \beta}
$$

we can write (10) in the form

$$
2 T_{F}=\sum_{\alpha, \beta=1}^{6} V_{\alpha} V_{\beta} F_{\alpha \beta} .
$$

Since $\nabla^{2} \varphi_{\alpha}=0$ for all $\alpha$,

$$
\int_{S} \varphi_{\alpha} \frac{\partial \varphi_{\beta}}{\partial n} d S=\int_{S} \varphi_{\beta} \frac{\partial \varphi_{\alpha}}{\partial n} d S
$$

so that $F_{\alpha \beta}=F_{\beta \alpha}$, and hence Eq. (12) involves 21 independent constants.

4. Equations of motion of the body. The total kinetic energy $T$ of fluid and body combined is

$$
T=T_{B}+T_{F}=\frac{1}{2} \sum_{\alpha, \beta} V_{\alpha} V_{\beta} A_{\alpha \beta},
$$

\footnotetext{
*Lamb, loc. cit.
} 
where $A_{\alpha \beta}=M_{\alpha \beta}+F_{\alpha \beta}$. The equations of motion of the body in terms of $T$ are:

$$
\begin{aligned}
& \frac{d}{d t} \frac{\partial T}{\partial V_{1}}-V_{6} \frac{\partial T}{\partial V_{2}}+V_{5} \frac{\partial T}{\partial V_{3}}=X_{1}, \\
& \frac{d}{d t} \frac{\partial T}{\partial V_{2}}-V_{4} \frac{\partial T}{\partial V_{3}}+V_{6} \frac{\partial T}{\partial V_{1}}=X_{2}, \\
& \frac{d}{d t} \frac{\partial T}{\partial V_{3}}-V_{5} \frac{\partial T}{\partial V_{4}}+V_{4} \frac{\partial T}{\partial V_{2}}=X_{3}, \\
& \frac{d}{d t} \frac{\partial T}{\partial V_{4}}-V_{3} \frac{\partial T}{\partial V_{2}}+V_{2} \frac{\partial T}{\partial V_{3}}-V_{6} \frac{\partial T}{\partial V_{5}}+V_{5} \frac{\partial T}{\partial V_{6}}=X_{4}, \\
& \frac{d}{d t} \frac{\partial T}{\partial V_{5}}-V_{1} \frac{\partial T}{\partial V_{3}}+V_{3} \frac{\partial T}{\partial V_{1}}-V_{4} \frac{\partial T}{\partial V_{6}}+V_{6} \frac{\partial T}{\partial V_{4}}=X_{5}, \\
& \frac{d}{d t} \frac{\partial T}{\partial V_{6}}-V_{2} \frac{\partial T}{\partial V_{1}}+V_{1} \frac{\partial T}{\partial V_{2}}-V_{5} \frac{\partial T}{\partial V_{4}}+V_{4} \frac{\partial T}{\partial V_{5}}=X_{6},
\end{aligned}
$$

where $X_{1}, X_{2}, X_{3}$ are the components of the external forces and $X_{4}, X_{5}, X_{6}$ are the components of the external moments with respect to 0 that are applied to the body. (By external forces and moments we mean forces and moments other than those due to the fluid pressures.)

5. Rectilinear motion. We now choose a special orientation for our coordinate axes, namely that of the three mutually perpendicular axes of "permanent translation" of the body. These have the property that if the body is set in motion parallel to one of them, without rotation and in the absence of external forces, it will continue to move in this manner; i.e., for motion with constant velocity in one of these directions the fluid exerts no force or moment on the body. We shall now proceed to examine the external forces and moments necessary for accelerated motion of the body in one of these directions.

It can be shown that if the directions of permanent translation are chosen as coordinate directions, the constants

$$
F_{12}=F_{13}=F_{23}=F_{21}=F_{31}=F_{32}=0 .
$$

In the following we shall always consider this choice to have been made.

6. Motion with velocity $V_{1}(t)$. Let us consider first the case when $V_{1}=V_{1}(t)$ and all other $V_{\alpha}=0$ for all time. Then the equations of motion (14) together with the expression for the kinetic energy (13) give:

$$
\begin{aligned}
& V_{1}^{\cdot} A_{11}=X_{1}, \quad V_{1}^{\cdot} A_{12}=X_{2}, \quad V_{1}^{\cdot} A_{13}=X_{3}, \quad V_{1}^{\cdot} A_{14}=X_{4}, \\
& V_{1}^{\cdot} A_{15}-V_{1}^{2} A_{13}=X_{5}, \quad V_{1}^{\cdot} A_{16}+V_{1}^{2} A_{12}=X_{6},
\end{aligned}
$$

where the dot denotes differentiation with respect to time. Hence, from (6) and (15)

$$
\begin{array}{ll}
X_{1}=V_{1}^{\cdot} A_{11}, & X_{2}=X_{3}=0, \\
X_{4}=r_{1}^{\cdot} A_{14}, & X_{5}=V_{1} A_{15}, \quad X_{6}=V_{1}^{\cdot} A_{16} .
\end{array}
$$


Expressions (17) represent the system of forces $X_{1}, X_{2}, X_{3}$ whose line of action passes through 0 , and the system of moments $X_{4}, X_{5}, X_{6}$ about 0 , which must be applied to the body so that it may move in the prescribed manner. Our object is to ascertain if there exists a point $0^{(1)}$ such that, if the line of action of the forces $X_{1}, X_{2}, X_{3}$ passes through it instead of through 0 , the external moments $X_{4}^{(1)}, X_{5}^{(1)}, X_{6}^{(1)}$ with respect to $\boldsymbol{0}^{(1)}$ will vanish. If such a point exists, then, under the sole action of the external force $X_{1}$ applied through $0^{(1)}$, (we may now conveniently call this force $X_{1}^{(1)}$ ), the body will always move without rotation and in the direction of the $x_{1}$ axis.

To investigate this question we refer all quantities to a new coordinate system whose origin is at a point $0^{(1)}$ and whose axes are parallel to those of the original system.

Let the velocities of the body referred to this new point be $V_{\alpha}^{(1)}$; and let $d_{1}^{(1)}, d_{2}^{(1)}$, $d_{3}^{(1)}$ be the components of the displacement of $0^{(1)}$ from 0 . Then

$$
\begin{aligned}
& V_{4}=V_{4}^{(1)}, \quad V_{5}=V_{5}^{(1)}, \quad V_{6}=V_{6}^{(1)}, \\
& V_{1}=V_{1}^{(1)}+V_{6}^{(1)} d_{2}^{(1)}-V_{5}^{(1)} d_{3}^{(1)}, \\
& V_{2}=V_{2}^{(1)}+V_{4}^{(1)} d_{3}^{(1)}-V_{6}^{(1)} d_{1}^{(1)}, \\
& V_{3}=V_{3}^{(1)}+V_{5}^{(1)} d_{1}^{(1)}-V_{4}^{(1)} d_{2}^{(1)} .
\end{aligned}
$$

If we substitute expressions (18) into the formula (13) for the kinetic energy and collect coefficients of $V_{\alpha}^{(1)} V_{\beta}^{(1)}$, which we call $A_{\alpha \beta}^{(1)}$, we obtain

$$
\begin{aligned}
& A_{14}^{(1)}=A_{14}+A_{12} d_{3}^{(1)}-A_{13} d_{2}^{(1)}, \\
& A_{15}^{(1)}=A_{15}+A_{13} d_{1}^{(1)}-A_{11} d_{3}^{(1)}, \\
& A_{16}^{(1)}=A_{16}+A_{11} d_{2}^{(1)}-A_{12} d_{1}^{(1)} .
\end{aligned}
$$

The remaining coefficients are not of interest at the moment.

If we now rewrite the equations of motion with respect to the $V_{\alpha}^{(1)}$ and $0^{(1)}$ we obtain equations identical with (17) except that all quantities will now have superscripts (1). Hence the external moments about $0^{(1)}$ can vanish only if

$$
A_{14}^{(1)}=A_{15}^{(1)}=A_{16}^{(1)}=0 \text {. }
$$

Using (19), (15) and (6) this means we must have:

$$
\begin{aligned}
& F_{14}=F_{14}^{(1)}=0, \\
& F_{15}+M_{15}-\left(F_{11}+M_{11}\right) d_{3}^{(1)}=0, \\
& F_{16}+M_{16}+\left(F_{11}+M_{11}\right) d_{2}^{(1)}=0 .
\end{aligned}
$$

Since $F_{14}$ does not vanish in general, equations (20) cannot generally be satisfied and hence there exists no point with respect to which the moment of fluid pressures vanishes for accelerated motion in the direction of the $x_{1}$ axis. It is clear that analogous conclusions will be reached for motion along the two other axes.

We may note that this result could have been deduced directly from Eqs. (17) referring to the original system. The components of the external force which can contribute to the moment $X_{4}^{(1)}$ are $X_{2}$ and $X_{3}$; but these vanish. Hence we must have $X_{4}=X_{4}^{(1)}$, i.e., the moment is the same for any reference point and is therefore due to 
a pure couple. Thus a change in the point of application of the external force can have no effect on $X_{4}$.

Returning to Eqs. (20), we see that if $F_{14}$ happens to vanish for a particular body, then all Eqs. (20) can be satisfied by choosing $0^{(1)}$ appropriately. Using (6) we then have:

$$
d_{3}^{(1)}=\frac{F_{15}+M x_{3}^{*}}{F_{11}+M}, \quad d_{2}^{(1)}=-\frac{F_{16}^{\prime}-M x_{2}^{*}}{F_{11}+M} .
$$

Hence in this case we can find a point $0^{(1)}$, or rather an axis $x_{1}^{(1)}$, (since $d_{1}^{(1)}$ is arbitrary), such that under the sole action of a force $X_{1}^{(1)}$ along this axis, the body will always move parallel to this axis and without rotation. We might call such an axis, an "axis of rectilinear translation".

The condition that $F_{14}=0$ imposes a restriction on the body shape and so might be regarded as a symmetry requirement.

If the original origin 0 is taken at the center of mass of the body then the terms $M x_{3}^{*}$ and $M x_{2}^{*}$ vanish in (21).

7. Motion with velocity $V_{2}(t)$. Repeating the above considerations for the case when all $V_{\alpha}$ except $V_{2}$ vanish for all time by transforming to a new system with origin at $0^{(2)}$; we find that the external moments about $0^{(2)}$ will vanish only if

$$
\begin{aligned}
F_{24}+M_{24}+\left(F_{22}+M_{22}\right) d_{3}^{(2)} & =0, \\
& =0, \\
F_{25} & =0 .
\end{aligned}
$$

As expected, these equations have no solution in the general case since $F_{25}$ is not generally zero.

If $F_{25}$ happens to be zero, Eqs. (22) are satisfied if

$$
d_{3}^{(2)}=-\frac{F_{24}-M x_{3}^{*}}{F_{22}+M}, \quad d_{1}^{(2)}=\frac{F_{26}+M x_{1}^{*}}{F_{22}+M} .
$$

Hence there exists an axis $x_{2}^{(2)}$ analogous to the axis $x_{1}^{(1)}$ found previously. We note, however, that the two axes will not, in general, intersect since $d_{3}^{(1)} \neq d_{3}^{(2)}$ except under special circumstances.

Since an analogous result will be obtained for motion with velocity $V_{3}$ we can sum up our results as follows:

(i) In general, rectilinear translational motion under the sole action of one external force is not possible.

(ii) If the body shape satisfies a certain symmetry condition, e.g., $F_{14}=0$ when referred to coordinate axes parallel to the axes of permanent translation, then there exists an axis of rectilinear translation for motion with velocity $V_{1}$. Similarly, if $F_{25}=0$ or $F_{36}=0$, there exist axes of rectilinear translation for motion with velocity $V_{2}$ or $V_{3}$, respectively.

(iii) In general no two of these axes, if more than one exists, will intersect.

8. Plane motion without circulation. If the body is an infinite cylinder with its generators parallel to the $x_{3}$ axis which moves with velocity components $V_{1}, V_{2}$ and $V_{6}$ only, then the fluid motion will take place in planes parallel to the $x_{1}, x_{2}$ plane and the two axes of permanent translation will lie in this plane. As before we choose our 
coordinate system in the direction of these axes. For such plane motion all coefficients $A_{\alpha \beta}$ in the expression (13) for the kinetic energy having one or both subscripts equal to 3,4 or 5 , vanish.

Considering motion with velocity $V_{1}$ only, we obtain equations (17) with $A_{14}=A_{15}=0$ and hence $X_{4}=X_{5}=0$. The transformation to a new coordinate system with origin $0^{(1)}$ yields one equation for $d_{2}^{(1)}$ which will make $A_{16}^{(1)}=0$. It is the same as the second Eq. (21). This determines one axis of rectilinear translation.

Similar arguments hold for motion with velocity $V_{2}(t)$ and they lead to the second Eq. (23) for $d_{1}^{(2)}$ which will make $A_{26}^{(2)}=0$. This determines the second axis of rectilinear motion.

Since both axes lie in the plane of motion, they intersect at a point $P$, say, with coordinates $x_{1}^{(a)}$ and $x_{2}^{(a)}$. If the origin of our first coordinate system is taken at the center of mass of the body, then $x_{1}^{(a)}$ and $x_{2}^{(a)}$ are given by the equations for $d_{1}^{(2)}$ and $d_{2}^{(1)}$, respectively, with $x_{1}^{*}=x_{2}^{*}=0$ :

$$
x_{1}^{(a)}=\frac{F_{28}}{F_{22}+M}, \quad x_{2}^{(a)}=-\frac{F_{16}}{F_{11}+M} .
$$

We might call this point the "apparent center of mass of the body", keeping in mind, however, that its position also depends on the density of the fluid which appears in the constants $F_{\alpha \beta}$.

It is interesting to consider pure rotation about this point with velocity $V_{6}(t)$. The equations of motion are found to be:

$$
\begin{gathered}
A_{16}^{(a)} V_{6}^{\cdot}-V_{6}^{2} A_{26}^{(a)}=X_{1}^{(a)}, \quad A_{26}^{(a)} V_{6}^{\cdot}+V_{6}^{2} A_{16}^{(a)}=X_{2}^{(a)}, \\
X_{3}=X_{4}=X_{5}=0, \quad A_{66}^{(a)} V_{6}^{\prime}=X_{6}^{(a)},
\end{gathered}
$$

where the superscript $(a)$ means that we are referring all quantities to a coordinate system whose axes are the axes of rectilinear motion and whose origin is therefore $x_{1}^{(a)}$, $x_{2}^{(a)}$. Since $A_{16}^{(a)}=A_{26}^{(a)}=0$ the only external force or moment on the body is a moment about the origin. Thus the point $x_{1}^{(a)}, x_{2}^{(a)}$ also acts as an apparent center of mass in the case of pure rotation about this point.

It is important to note that the motions and corresponding forces and moments considered above cannot be simply superimposed. A translation of the apparent center of mass, for example, with velocity components $V_{1}$ and $V_{2}$ but without rotation, cannot occur without the action of an external moment about the apparent center of mass. This is due to the non-linearity of the problem. The analogy between the apparent center of mass for motion of a body in a fluid and the center of mass is restricted to three particular motions, the two translations of the body parallel to the axes of permanent (or rectilinear) translation, and rotation about the apparent center of mass.

9. Body of revolution. The axis of revolution is a direction of permanent translation. Let it be the $x_{1}$ axis. For motion in the $x_{1}$ direction we consider Eqs. (20). For a body of revolution $F_{14}$ must always be zero because changing the sign of $V_{4}$ in the expression for the kinetic energy must leave the latter unchanged. Similarly $F_{15}=F_{16}=0$; also $M_{15}=M x_{3}^{*}=0$ and $M_{16}=-M x_{2}^{*}=0$ since the center of mass lies on the axis of revolution; hence $d_{3}^{(1)}=d_{2}^{(1)}=0$. This gives the obvious result that the axis of rotation is an axis of rectilinear translation.

From symmetry considerations it is clear that the choice of directions for the axes 
$x_{2}$ and $x_{3}$ is immaterial. For motion with velocity $V_{2}$, say, we must consider Eqs. (22). Applying symmetry considerations to the expression for $T$, as was done above, we find that $F_{24}=F_{25}=0$. Hence

$$
\begin{aligned}
& A_{24}^{(2)}=M_{24}+\left(F_{22}+M_{22}\right) d_{3}^{(2)}=0 \\
& A_{26}^{(2)}=F_{26}+M_{26}-\left(F_{22}+M_{22}\right) d_{1}^{(2)}=0 .
\end{aligned}
$$

But

$$
M_{24}=-M x_{3}^{*}=0 .
$$

Thus $d_{3}^{(2)}=0$, and there remains

$$
d_{1}^{(2)}=\frac{F_{26}+M x_{1}^{*}}{F_{22}+M},
$$

which defines an axis of rectilinear translation.

For motion with velocity $V_{3}$ we obtain in the same manner $d_{2}^{(3)}=0$ and

$$
d_{1}^{(3)}=-\frac{F_{35}-M x_{1}^{*}}{F_{33}+M} .
$$

From the symmetry of the body it is seen that $F_{22}=F_{33}$ and $F_{26}=-F_{35}$. Hence $d_{1}^{(2)}=d_{1}^{(3)}$ as was to be expected from our assertion that the choice of the directions $x_{2}$ and $x_{3}$ was immaterial.

Thus, as in the case of plane motion, there exists a center of apparent mass, but here there are infinitely many axes of rectilinear motion: the axis of revolution and any axis perpendicular to the latter and passing through the apparent center of mass.

Let us consider a motion of pure rotation about an axis of rectilinear motion. Clearly, for motion with $V_{4}(t)$, i.e., rotation about the axis of revolution, the only force or moment is

$$
X_{6}^{(a)}=M_{44}^{(a)} V_{4}^{\cdot} .
$$

For motion with velocity $V_{6}$, say, about the appropriate axis of rectilinear motion we have:

$$
\begin{array}{ll}
A_{16}^{(a)} V_{6}^{\cdot}-V_{6}^{2} A_{26}^{(a)} & =X_{1}^{(a)}, \\
A_{26}^{(a)} V_{6}^{\cdot}+V_{6}^{2} A_{16}^{(a)} & =X_{2}^{(a)}, \\
A_{36}^{(a)} V_{6}^{\cdot} & =X_{3}^{(a)}, \\
A_{46}^{(a)} V_{6}^{\cdot}-V_{6}^{2} A_{56}^{(a)} & =X_{4}^{(a)}, \\
A_{56}^{(a)} V_{6}^{\cdot}+V_{6}^{2} A_{46}^{(a)} & =X_{5}^{(a)}, \\
A_{66}^{(a)} V_{6}^{\cdot} & =X_{6}^{(a)} .
\end{array}
$$

But $A_{16}^{(a)}=A_{26}^{(a)}=0$ and $A_{36}^{(a)}, A_{48}^{(a)}, A_{58}^{(a)}$ vanish from symmetry considerations. Hence all forces and moments with the exception of $X_{6}^{(a)}$ vanish. Thus the concept of the apparent center of mass for a body of revolution is also valid for pure rotation about an 
axis of rectilinear motion in general. An exception to this is the motion due to a combination of velocities $V_{1}(t)$ and $V_{2}(t)$.

As in the plane case, however, simple superposition of the special motions and forces discussed is not permissible.

It should be noted that, even if all assumptions made in this analysis are sufficiently well justified, some of the special motions discussed cannot be expected to be observed in nature because they are unstable when subjected to small disturbances. 